EPJ Web of Conferences 18, 03003 (2011)

DOI: $10.1051 /$ epjconf/20111803003

(C) Owned by the authors, published by EDP Sciences, 2011

\title{
Theoretical approaches to nucleation in the gas phase. With a glimpse at experiments and dust formation in astrophysics
}

\author{
P. Parneix ${ }^{1}$ and F. Calvo ${ }^{2}$ \\ 1 Institut des Sciences Moléculaires d'Orsay, CNRS UMR 8214, Université Paris Sud 11, \\ Bât. 210, 91405 Orsay, France \\ 2 LASIM, CNRS and Université Claude Bernard Lyon I, 43 Bd. du 11 Novembre 1918, \\ 69622 Villeurbanne Cedex, France
}

\begin{abstract}
An overview of the physics of homogeneous nucleation is presented, focusing on the main theoretical approaches that are currently available. We review the microscopic assumptions behind classical nucleation theory, as well as several improvements with various degrees of empiricism. The increasing role played by molecular simulations in testing and correcting nucleation theories is discussed. Typical laboratory experiments on homogeneous nucleation are also reviewed, emphasizing how experimental observables can be compared with theoretical predictions. Finally, the more specific challenges of nucleation in astrophysical media are discussed.
\end{abstract}

\section{INTRODUCTION}

Nucleation is an out-of-equilibrium process in which a new phase is created from a different metastable phase. Nucleations of condensed phases are critical phenomena that drastically depend on external thermodynamic parameters, especially pressure and temperature. Nucleation occurs in a broad variety of natural processes such as crystal growth, bubble formation in liquids (cavitation), aerosol and cloud formation in planetary atmospheres [1,2], as well as in combustion and flames [3-5]. As will be discussed below in more details, nucleation processes are also fundamentally important in astrophysics and astrochemistry.

The formation of liquid droplets in a supersaturated vapor is archetypal of nucleation in the context of the liquid/vapor phase transition. From the microscopic point of view, it conveys the growth of atomic or molecular clusters surrounded by a supersaturated gas containing mostly monomers and that tend to stick to the droplet more rapidly than the reverse evaporation process. Homogeneous nucleation, in which the clusters are formed from a pure vapor, should be distinguished here from heterogeneous nucleation where cluster growth is triggered by a surface or, in a volume, by the presence of impurities. However, even though they are of greater practical importance [6], the latter processes involve much more complex mechanisms. In the present paper, only homogeneous nucleation will be described and discussed.

The conventional view of nucleation from an homogeneous phase involves the decay of the metastable phase (supersaturated vapor) into the localized formation of droplets (liquid phase), through a free-energy barrier that depends on the external thermodynamical conditions — and vanishes above the critical temperature. This barrier located at a so-called critical nucleus separates small clusters that evaporate faster than they grow, from larger droplets that grow continuously. The critical nucleus acts as a bottleneck for cluster growth, and the shape of the barrier, in particular, affects the kinetics with which critical droplets will, ultimately, either grow or shrink.

This is an Open Access article distributed under the terms of the Creative Commons Attribution-Noncommercial License 3.0, which permits unrestricted use, distribution, and reproduction in any noncommercial medium, provided the original work is properly cited. 


\section{EPJ Web of Conferences}

Classical nucleation theory (CNT), described in details below, is built following the lines of the above macroscopic picture. CNT is by far the most widespread approach for describing homogeneous nucleation, especially in the context of interpreting measurements as well as computer experiments. However, it lacks microscopic input and completely neglects information about interactions, and may thus be plagued by the breakdown of macroscopic concepts at small sizes well known in cluster physics as the non-scalable regime [7]. Only recently, new theoretical frameworks have been proposed in order to test the underlying hypotheses of classical nucleation theories, and to improve them by including more atomistic data. The field has particularly benefited from progresses in molecular simulation, which together with advances in the modeling of intramolecular forces have lead to much deeper understanding of nucleation phenomena.

The next section is devoted to a critical discussion of CNT, including its basic formalism, some known flaws and various improvements. Several important developments related to the computation of free energies are also presented, including classical density functional theory (DFT) and dynamical nucleation theory (DNT). In Sec. 3 we briefly look at experimental techniques used in the laboratory to analyse nucleation kinetics. Only in Sec. 4, and based on the concepts discussed in the preceding sections, the importance of nucleation in astrophysics and astrochemistry will be highlighted.

\section{THEORIES OF NUCLEATION}

Nucleation theories seek to characterize the equilibrium state of a cluster which can exchange atoms with the surrounding vapor, depending on the physical parameters (temperature, density, etc.), as well as the related out-of-equilibrium quantities, namely the evaporation and condensation rates.

In this section we present some of the most widely used theoretical methods for describing nucleation of condensed droplets from the gas phase. This introduction is mainly aimed at providing the basic concepts, limitations and improvements of the broad frameworks of classical nucleation theory and density functional theory, and also to give some elements of recent progresses on the validation of these macroscopic models from the microscopic or molecular points of view.

\subsection{Main assumptions and steady-state hypothesis}

Nucleation theories are essentially kinetic approaches that rely on elementary mechanisms by which the clusters gain or lose particles, focusing on their associated rates and the equilibrium distribution of cluster sizes at specific external conditions. The various populations of cluster sizes evolve through a set of coupled linear equations that convey their growth or loss. This immediately entails several difficulties. First, standard statistical theories such as CNT below predict that the rates are usually only dependent on cluster size and temperature, thus they neglect the variations in temperature upon dissociation or growth; such effects will only be negligible for large clusters. Also, such a treatment implies that the clusters can be unambiguously defined from their surrounding vapor, which raises some issues near the critical temperature where fluctuations can be huge.

It is useful to start from a microscopic perspective, by considering the equilibrium between the gaz and liquid phases in a restricted volume, assuming this volume contains clusters of various sizes and a surrounding vapor consisting mostly of monomers. This point of view is fundamental for classical nucleation theory, but has also been adopted in early computational investigations [8], followed by many others [9-12], for its relevance in many areas and primarily in atmospheric science. At equilibrium, the homogeneous nucleation equilibrium can be summarized by the reactions

$$
\mathrm{X}_{n+1} \rightleftharpoons \mathrm{X}_{n}+\mathrm{X}
$$

for different cluster sizes $n$. The kinetics of this set of reactions can be modelled using a master equation, whose solution requires knowledge of the condensation $\left(k_{\mathrm{cond}}\right)$ and evaporation $\left(k_{\mathrm{evap}}\right)$ rates. The net rate 
$k_{n}(T)$ at which clusters of size $n$ become clusters of size $n+1$ is expressed as

$$
k_{n}(T)=k_{\text {cond }}(n) \mathcal{P}(n, T)-k_{\text {evap }}(n+1) \mathcal{P}(n+1, T)
$$

where $\mathcal{P}(n, T)$ is the concentration of clusters with size $n$ at temperature $T$. At equilibrium, all rates share a common value called the nucleation rate $k_{\mathrm{ss}}(T)$, where the subscript ss indicates the steady-state approximation. Under such conditions, the condensation and evaporation fluxes are equal and the rates are related to each other through the equilibrium concentrations $\mathcal{P}^{\text {eq }}(n, T)$

$$
\frac{k_{\text {cond }}(n)}{k_{\text {evap }}(n+1)}=\frac{\mathcal{P}^{\mathrm{eq}}(n+1, T)}{\mathcal{P}^{\mathrm{eq}}(n, T)} .
$$

After a few manipulations, inserting the previous relation into Eq. (2) leads to the expression of the nucleation rate as a function of only condensation or evaporation rate constants:

$$
\begin{aligned}
k_{\mathrm{ss}}(T) & =\mathcal{P}(1)\left[\sum_{n \geq 1} \frac{\mathcal{P}^{\mathrm{eq}}(1, T)}{k_{\text {cond }}(n) \mathcal{P}^{\mathrm{eq}}(n, T)}\right]^{-1} \\
& =\mathcal{P}(1)\left[\sum_{n \geq 1} \frac{\mathcal{P}^{\mathrm{eq}}(1, T)}{k_{\text {evap }}(n+1) \mathcal{P}^{\mathrm{eq}}(n+1, T)}\right]^{-1} .
\end{aligned}
$$

At this stage, additional approximations are needed to evaluate the nucleation rate in practice.

\subsection{Classical nucleation theory}

Classical nucleation theory [13-15] relies on macroscopic thermodynamical concepts and considers the equilibrium between a spherical liquid droplet having $n$ atoms and a supersaturated vapor of the same element. CNT assumes that the interface between the liquid and the vapor is sharp (capillary approximation), and that the droplet shares the properties of the bulk liquid. The equilibrium probability of forming a cluster with $n$ atoms from the vapor depends on the supersaturation ratio $S=p / p_{\text {sat }}$, where $p_{\text {sat }}$ is the saturation pressure and $p$ the gas pressure, respectively. $\mathcal{P}^{\mathrm{eq}}(n, T)$ is proportional to $S^{n-1}$, and also to $\exp \left(-\Delta G(n) / k_{\mathrm{B}} T\right)$, where $\Delta G(n)$ is the Gibbs free energy change for the reaction bringing the $n$ atoms from the vapor into the condensed phase. In the basic version of CNT (Dillmann-Meier theory), $\Delta G(n)$ has the following expression

$$
\Delta G(n)=n\left(\mu_{\ell}-\mu_{v}\right)+4 \pi R^{2} \sigma,
$$

where $\mu_{v}$ and $\mu_{\ell}$ stand for the chemical potential of the vapor and liquid phases, respectively, $\sigma$ is the surface tension and $R \propto n^{1 / 3}$ is the droplet radius. If we consider the metastable transformation leading to the formation of the liquid droplet from the supersaturated vapor at constant temperature, the volumic contribution $n\left(\mu_{\ell}-\mu_{v}\right)$ can be expressed by integrating along an isotherm from the saturation pressure $p_{\text {sat }}$, assimilitating the vapor as a perfect gas. This calculation leads to

$$
n\left(\mu_{\ell}-\mu_{v}\right)=n k_{\mathrm{B}} T \ln p / p_{\text {sat }}=\frac{4}{3} \pi R^{3} \rho k_{\mathrm{B}} T \ln S,
$$

where we have introduced the liquid density $\rho$.

By definition, the critical radius $R^{*}$ corresponds to the maximum of the Gibbs free energy, also called the nucleation barrier. This quantity is essential in all nucleation theories. Differentiating the expression of $\Delta G$ as a function of $R$, the solution of $\partial \Delta G / \partial R=0$ is found at $R^{*}$ such that

$$
R^{*}=\frac{2 \sigma}{\rho k_{\mathrm{B}} T \ln S} .
$$




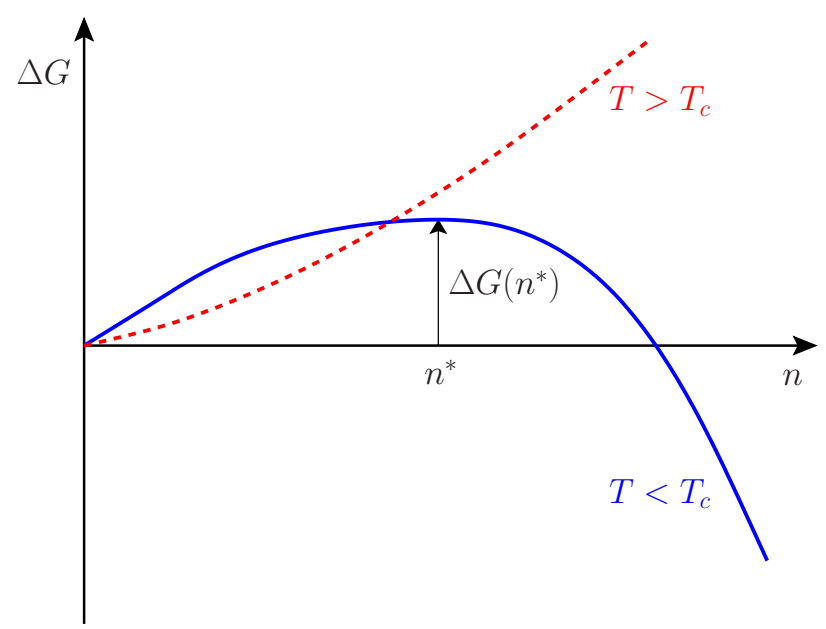

Figure 1. Schematic variations of the free energy corresponding to the formation of a cluster from the vapor, as a function of its size. Below the critical temperature, the liquid can grow into a cluster provided that a barrier $\Delta G\left(n^{*}\right)$ at the critical nucleus $n=n^{*}$ is crossed. Above the critical temperature, only the vapor is stable.

This last equation, known as the Kelvin relation, is often used to relate external thermodynamic parameters (pressure and density) to the physical properties of critical droplets. In particular, it emphasizes that CNT is fundamentally a macroscopic approach, which does not rely on molecular data.

The above arguments hold at temperatures below the critical temperature $T_{c}$. At $T>T_{c}$, only the vapor phase is stable, $\mu_{v}<\mu_{\ell}$ and the nucleation barrier vanishes (see Fig. 1). The next step is to simplify the expressions of Eqn. (4) and (5) for the nucleation rate, by keeping only the term which contributes most to the infinite sums, that is the one with the lowest denominator. Precisely, this term is obtained at the critical radius $n=n^{*}$, for which $\Delta G$ is maximum. Therefore $k_{\mathrm{SS}}$ is determined by a single Arrhenius relation involving the nucleation barrier, equal to $\Delta G$ at $R=R^{*}$ or the corresponding $n=n^{*}$ :

$$
k_{\mathrm{ss}}=k_{0} \exp \left(-\frac{\Delta G\left(n^{*}\right)}{k_{\mathrm{B}} T}\right)
$$

where the prefactor $k_{0}$ will be discussed below. Inserting the Kelvin relation of Eq. (8) into the expression of the Gibbs free energy yields the expression of free energy barrier as

$$
\Delta G\left(n^{*}\right)=\frac{16 \pi}{3} \frac{\sigma^{3}}{\left[\rho k_{\mathrm{B}} T \ln S\right]^{2}},
$$

from which we finally get the expression of nucleation rate in the the steady-state approximation [14, 15]

$$
k_{\mathrm{ss}}=k_{0} \exp \left[-\frac{16 \pi \sigma^{3}}{3 \rho^{2}\left(k_{\mathrm{B}} T\right)^{3}(\ln S)^{2}}\right] .
$$

The prefactor $k_{0}$ is expressed in CNT as the product of two independent contributions, $k_{0}=k^{*} \times \Gamma$, where $k^{*}$ is the attachment rate to critical droplets and $\Gamma$ is the so-called Zel'dovich factor, which captures multiple recrossings of the free-energy barrier [16]:

$$
\Gamma=\left(\frac{\eta}{2 \pi k_{\mathrm{B}} T}\right)^{1 / 2}, \eta=-\left.\frac{\partial^{2} \Delta G}{\partial n^{2}}\right|_{n=n^{*}} .
$$

The various approximations involved in classical nucleation theory play on rather different levels. The expression of the nucleation rate as an exponential function of a free-energy barrier, Eq. (9), can be 
obtained by modeling the time evolution of the population of cluster sizes by a one-dimensional Markov chain [14], using the steady-state solution for the nucleation rate. The expression of the free energy as a function of cluster size, $\Delta G(n)$, is a very crucial ingredient as well, for which Eq. (6) is only a crude, first-order representation. The Zel'dovich factor, on the other hand, should be considered as a correcting term.

Due to the various parameters involved, using the previous equations for interpreting experimental data is not straightforward. The two control parameters are the temperature $T$ and supersaturation ratio $S$, whereas the measured quantity is the nucleation rate $k$. Several authors have tried to uncover additional relations between these three quantities, leaving other unkown data to be fitted. The "nucleation theorems" derived by Kashchiev [17, 18] and reformulated by other authors [19, 20], for example, use the following general expression as a template for the nucleation rate $k(T, S)$ :

$$
k(T, S)=A S^{c} \exp \left[-\frac{B}{(\ln S)^{2}}\right],
$$

where $A$ and $B$ are adjustable parameters, $c$ is taken as 1 (CNT would strictly predict $c=2$ ). The first nucleation theorem, in particular, relates the critical size $n^{*}$ to the variations of the nucleation rate with supersaturation ratio at fixed temperature $T$ as $[17,18]$

$$
\left.n^{*} \approx \frac{\partial \ln k}{\partial \ln S}\right|_{T}
$$

Based on classical nucleation theory, Hale proposed a model to relate the supersaturation ratio to the temperature, assuming that the surface tension $\sigma$ varies linearly with temperature and vanishes at the critical point [21] according to $\sigma(T)=\Omega\left(T_{c}-T\right)$. It is useful to introduce a critical supersaturation $S_{c}$ at which the experimental nucleation rates become detectable, that is when they reach the order of $1 \mathrm{~cm}^{-3} \mathrm{~s}^{-1}$. If the prefactor $k_{0}$ does not vary much with temperature, which is a reasonable approximation in most cases, the critical supersaturation is expressed as a simple function of temperature

$$
\ln S_{c}(T) \propto\left[\Omega\left(T_{c} / T-1\right)\right]^{3 / 2},
$$

This relation is extensively used for interpreting experimental data.

\subsection{Validation of classical nucleation theory}

As will be discussed below, CNT performs rather poorly for reproducing the experimental measurements on atomic and molecular fluids. Besides the major challenges in conducting accurate experiments, it is important to question the fundamental validity of classical nucleation theory, by treating separately its various assumptions. In particular, the Arrhenius expression for the nucleation rate already suggests that the largest discrepancies should arise from small errors in the free energies, rather than inacurracies in the prefactors.

A suitable approach for testing the hypotheses underlying CNT is to use a simple model exhibiting all features relevant to nucleation, without the complications entailed by intermolecular interactions. The Ising model [22] on square lattices is such a model, in which nucleation is practically driven by a very small external magnetic field. In this model, spins up and down play the role of liquid and gaseous atoms, respectively. Ising models have been extensively used in the context of nucleation physics. However, until recently [23], the conclusions reached by various authors were somewhat contradictory [24-27].

The Ising model is computationally efficient, but also simple enough to allow for several analytical evaluations of some of its statistical properties at finite temperature. The CNT assumptions to be questioned are

1. The Arrhenius expression connecting the nucleation rate to a free energy barrier at the critical nucleus, Eq. (9);

2. The simple expression for $\Delta G$ at the critical nucleus, Eq. (6). 


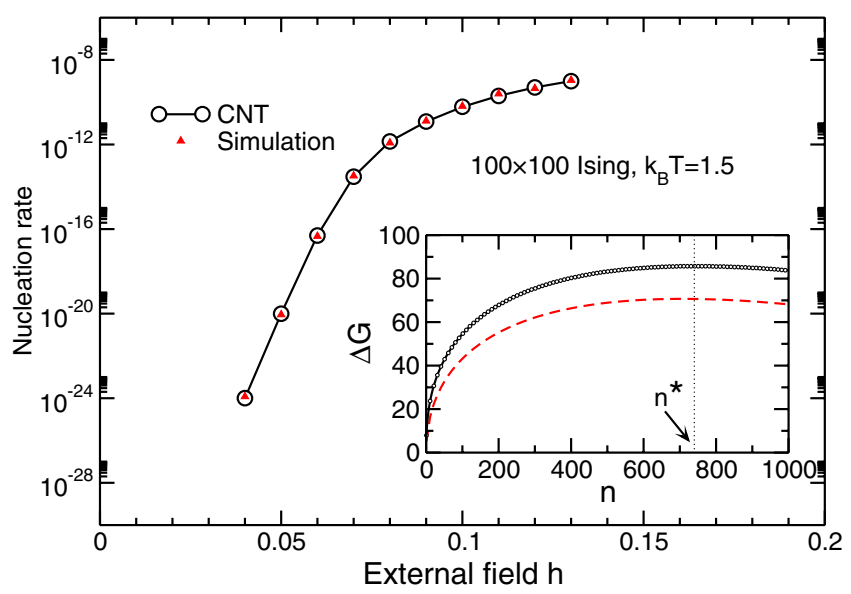

Figure 2. Nucleation rate in the 2D Ising model as a function of external magnetic field, as obtained from direct path sampling simulations (red triangles), or from classical nucleation theory with the free energy barrier $\Delta G\left(n^{*}\right)$ from Eq. (17). The inset shows the variations of $\Delta G$ with size $n$ from this equation (solid black line), from the simpler model of Eq. (16) (dashed red line), and from umbrella sampling Monte Carlo simulations (circles, essentially superimposed with the solid line). Data taken from Ryu and Cai [23].

It is more convenient to address the second assumption first, choosing the 2D Ising model as our example. The basic expression for the free energy at the critical nucleus, adapted to two dimensions, reads

$$
\Delta G(n)=\sigma n^{1 / 2}-2 h n
$$

where $h$ is the external electric field, playing the role of the difference in chemical potential in conventional nucleation theory. But it turns out that analytical predictions can also be made, leading to a more accurate expression similar to the phenomenological Fisher model [28]:

$$
\Delta G(n)=\sigma n^{1 / 2}-2 h n+\tau k_{\mathrm{B}} T \ln n+d(T),
$$

where $\tau=5 / 4$ and $d(T)$ is a constant only dependent on temperature. Alternatively, it is possible to compute the free energy profile by simulation through a series of umbrella sampling Monte Carlo simulations in which $\Delta G$ is built piece by piece around increasing values of $n$. While the results of these simulations agree with the latter expression of Eq. (17), they are strongly underestimated by the simpler form of Eq. (16). These differences are illustrated as an inset in Fig. 2.

Progresses in computational methodology also allow the nucleation rate to be directly obtained, using for instance transition path sampling [29]. It is then possible to compare the numerically accurate nucleation rate $k_{\text {cond }}(T)$ with the predictions of CNT, using the appropriate expression for the free energy barrier $\Delta G\left(n^{*}\right)$ and the Zel'dovich factor $\Gamma$. The nucleation rates computed by Ryu and Cai for the 2D Ising model at several values of $h$, shown in Fig. 2, match very well (within $50 \%$ error) those obtained from CNT, provided that the correct barrier height $\Delta G\left(n^{*}\right)$ is used. If the basic, two-parameter expression of Eq. (16) is employed instead, the rates are too high by orders of magnitude. These results are not specific to the $2 \mathrm{D}$ case, but hold as well in $3 \mathrm{D}$, with a modified expression for $\Delta G(n)[23]$.

The conclusion of this investigation is that classical nucleation theory is actually quantitative, once the free energy barrier is accurately determined. Unfortunately, the calculation of free energies is generally a truly challenging task in realistic molecular systems, because the interactions can be much more complicated than in the Ising model. In practice, even for simple atomic fluids such as argon, 
additional problems come into play that do not have any counterpart in lattice-based models. It is then tempting to further improve CNT through its most crucial thermodynamical ingredient, the free energy at the nucleation barrier.

\subsection{Phenomenological improvements to CNT}

Quantitative failure of CNT being largely due to the approximations involved in $\Delta G$, several authors have tried to correct for the most salient defficiencies of the Dillmann-Meier expression involving only the terms proportional to $n$ (volume contribution) and $n^{2 / 3}$ (surface contribution). We describe here several of these extensions and modifications, a more detailed discussion can be found in Ref. [30].

A first correction concerns the role of free translations, which contribute to the global Gibbs free energy as prefactors in the partition functions [31,32]. The conservation of the centre of mass of the droplet, also referred to as law of mass action, reduces the effective number of degrees of freedom in the cluster by 3, which amounts to reducing $n$ by $n-1$ in the first term of the right-hand-side of Eq. (6). Another phenomenological extension of CNT, called internal consistency, consists in replacing $n^{2 / 3}$ in the surface energy of the droplet by $n^{2 / 3}-1$, as an attempt to correct for the behavior at $n \rightarrow 1$ [33]. Both these modifications lead to minor improvements.

One major criticism to classical nucleation theory applied for small systems is that it is a macroscopic approach $[12,34]$. In this respect, the assumptions that the cluster shape is perfectly spherical, that its energy only depends on the volume and the surface, and that the surface tension does not depend on size can be questioned. In the spirit of the liquid drop model, the Gibbs free energy can be corrected using an expansion in powers of $n^{1 / 3}$ [35]. The dependence of surface tension on size occurs through a curvature effect as predicted by Tolman [36]

$$
\sigma(R)=\frac{\sigma(\infty)}{1+2 \delta / R}
$$

but this corrective term could also be accounted for by the liquid drop model, through a Taylor expansion [37]. Surface corrections have been shown to improve significantly the predictions of classical nucleation theory with respect to numerical calculations, even though no quantitative agreement with experiment could still be reached in the case of argon [38].

\subsection{Density-functional theory}

The improvements of CNT discussed previously are essentially a posteriori corrections to macroscopic quantities, but do not yet incorporate any information about the atomic interactions or the structure of the nucleus. One step in this direction, which is still on a macroscopic level but with insight from the interactions, is provided by classical density-functional theory [39]. The main motivation behind DFT is to bypass the capillarity approximation, which is essential in CNT, by computing the density profile of the liquid droplet in contact with the vapor under specific thermodynamical conditions, and for realistic interactions between the constituent molecules.

Density-functional theory applies to simple fluids with pair interactions that can be written as the sum of a mainly repulsive part $V_{0}(r)$ plus a perturbative attractive part $V(r)$. The Helmholtz free energy $F$ relatively to its value for the reference system $V_{0}$ is expressed by linear integration along a path joining the two fluids:

$$
F[\rho]=\frac{1}{2} \int_{0}^{1} d \alpha \iint d \mathbf{r} d \mathbf{r}^{\prime} \rho_{\alpha}\left(\mathbf{r}, \mathbf{r}^{\prime}\right) V_{\alpha}\left(\left|\mathbf{r}-\mathbf{r}^{\prime}\right|\right) .
$$

In this equation, and at a given point $\alpha$ along the path, the particles interact via the pair potential $V_{\alpha}(r)=\alpha V(r)+V_{0}(r)$, and $\rho_{\alpha}\left(\mathbf{r}, \mathbf{r}^{\prime}\right)$ is the pair distribution function corresponding to the intermediate potential $V_{\alpha}$. There are several difficulties in applying DFT for the nucleation problem [40]. The pair 


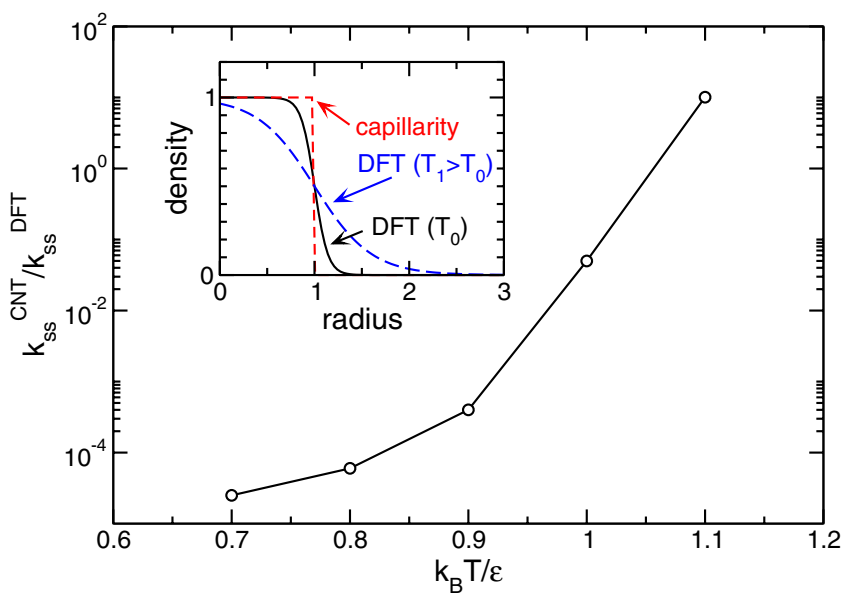

Figure 3. Ratio of nucleation rates for a Lennard-Jones fluid between the value obtained by classical nucleation theory and the predictions of density functional theory, as a function of temperature (data taken from Ref. [40]). The inset shows the smooth density profiles obtained for the liquid clusters at two temperatures, compared to the sharp profile resulting from the capillarity approximation in CNT.

distribution function can be decoupled using a random phase approximation, $\rho_{\alpha}\left(\mathbf{r}, \mathbf{r}^{\prime}\right) \simeq \rho_{\alpha}(\mathbf{r}) \times \rho_{\alpha}\left(\mathbf{r}^{\prime}\right)$. Also, for hard-sphere potentials, the reference free energy is satisfactorily approximated using the localdensity approximation.

The equilibrium density profile is determined from a variational condition, $\delta \Omega / \delta \rho(\mathbf{r})=0$, where $\Omega$ is the grand potential of the system. The solution of this equation, which is obtained iteratively, shows that the liquid/vapor interface is not sharp but increasingly smooth as temperature increases [40]. It is sketched as an inset in Fig. 3. The way to define the droplet radius under such conditions has been discussed by Talanquer and Oxtoby [41]. As shown by Zeng and Oxtoby [40], the free energies obtained from density-functional theory lead to comparable variations in the nucleation rates with respect to CNT. However the temperature dependence varies more significantly. This is illustrated in Fig. 3 for the Lennard-Jones fluid, where the ratio $k_{\mathrm{ss}}^{\mathrm{CNT}} / k_{\mathrm{ss}}^{\mathrm{DFT}}$ between the nucleation rates predicted by the two theories (with the exact same prefactor) shows significant variations with temperature.

The results obtained by classical DFT have inspired several attempts at improving classical nucleation theories by revisiting the capillarity approximation. The diffuse interface method of Gránásy [42], in particular, parametrizes the cross-interfacial free energies obtained for smooth profiles. Densityfunctional theory was also extended to treat ion-induced nucleation [43, 44], in order to address the controversial 'sign preference' issue [45] which states that condensation on negative anions is promoted over nucleation on cations.

\subsection{Molecular approaches and simulations}

Classical nucleation theories are designed from the perspective of a mesoscopic fluid droplet in contact with a supersaturated vapor, and entirely builds on macroscopic thermodynamics. While densityfunctional theory incorporates some elements of chemistry through the intermolecular interactions, it still uses the standard kinetic concepts of CNT in the prefactors.

The use of molecular simulation in nucleation theories dates back to Lee and coworkers [8], who attempted to calculate the free energies of clusters in contact with their own vapor. One major issue in computational works is concerned with the definition of a bound cluster. A widely used definition proposed by Stillinger [46] relies on geometry only, one atom being considered as part of a given cluster 
if it lies within some distance of any other atom from the cluster. This definition has been criticized by many authors, who came up with more sophisticated alternatives that take dynamical effects into account $[47,48]$.

Once a definition is chosen, it is possible to calculate the free energy profile with the same techniques used for the Ising model, such as umbrella sampling or other algorithms. Besides the free energies, the rates themselves can be estimated owing to progresses in computational techniques, either by direct molecular dynamics simulations, or more accurately through the recent algorithms proposed for sampling rare events. We discuss below one important framework developed for nucleation studies at the molecular level, namely dynamical nucleation theory. This approach, developed by Schenter and coworkers [49-51], is particularly appealing as it builds upon Eq. (5) to determine the nucleation rate, by calculating the evaporation rates of a series of cluster sizes. Thus the point of view of the dissociating cluster is adopted, in a way quite similar to microreversible statistical theories of fragmentation such as the Weisskopf [52] or Rice-Ramsperger-Kassel [53-55] models. Since DNT is an equilibrium approach, it requires many ingredients to be calculated from molecular simulations at equilibrium. The main originality of DNT is the use of variational transition state theory $[56,57]$ to compute the evaporation rate $k_{\text {evap }}(n)$ for a $n$-particle cluster as [49-51]

$$
k_{\text {evap }}^{\mathrm{TST}}(n) \propto-\frac{d F_{n}(\mathcal{V}, T)}{d R_{\text {cut }}},
$$

in which $F_{n}(\mathcal{V}, T)$ is the Helmholtz free energy of a $n$-particle cluster kept within volume $\mathcal{V}=4 \pi R_{\text {cut }}^{3} / 3$ and at canonical temperature $T$. The free energy is calculated by restricting configurations to remain within the sphere of radius $R_{\text {cut }}$. According to transition state theory, the dividing surface should be chosen at the value where the reactive flux is minimized [56,57]. This again is a dynamical definition of a cluster volume, at variance with the purely thermodynamical definition of classical nucleation theories. However, it should be stressed that its calculation remains based on equilibrium thermodynamics, no dynamical simulation of the evaporation process being actually performed at any stage. Independent simulations of thermal dissociation in clusters have shown that such purely statistical approaches may be in error by about one order of magnitude for the prefactors [58-61].

Helmholtz free energies play in DNT the same role as the Gibbs free energies in CNT, and they can be used to provide mass distributions at equilibrium [8] that in turn allow an estimation of the condensation rates by application of Eq. (3). In DNT, the free energies and mass distributions are obtained from statistical mechanics and Monte Carlo simulations [49-51]. When applied to realistic molecular systems, this immediately raises the problem of the accuracy of the intermolecular interactions. This problem is especially acute in the crucially important case of water, for which no potential energy function widely stands out. This is manifested on the different values for the evaporation and condensation rates of small water clusters when those are described using either a simple nonpolarizable (TIP4P) potential or the more accurate Dang-Chang model [62]. Very recently, the method could even be applied using ab initio potential energy surfaces [63].

However, while these issues are utterly relevant when attempting to compare with experimental results, they do not call into question the use of simulation as a benchmark for theories. As was done for the Ising model above, simulation can be used to calculate the ingredients needed in these theories, and to estimate the 'numerically exact' nucleation rates. In this respect, dynamical nucleation theory performs quite well, considering that the free energies are more difficult to extract than in the simple lattice-based Ising models. This is illustrated in Fig. 4, where the evaporation rates for water clusters containing up to a few tens of molecules, as predicted by the theory, vary similarly as the results from MD trajectories within a factor 2-5 [64].

Another appealing feature of computer simulations is that they allow to get insight into the condensation and evaporation processes, and even determine their individual rate constants, providing a stringent testing ground for nucleation theories for the same model. Interestingly, molecular dynamics trajectories reveal that monomers colliding on thermalized clusters do not always lead to condensation 


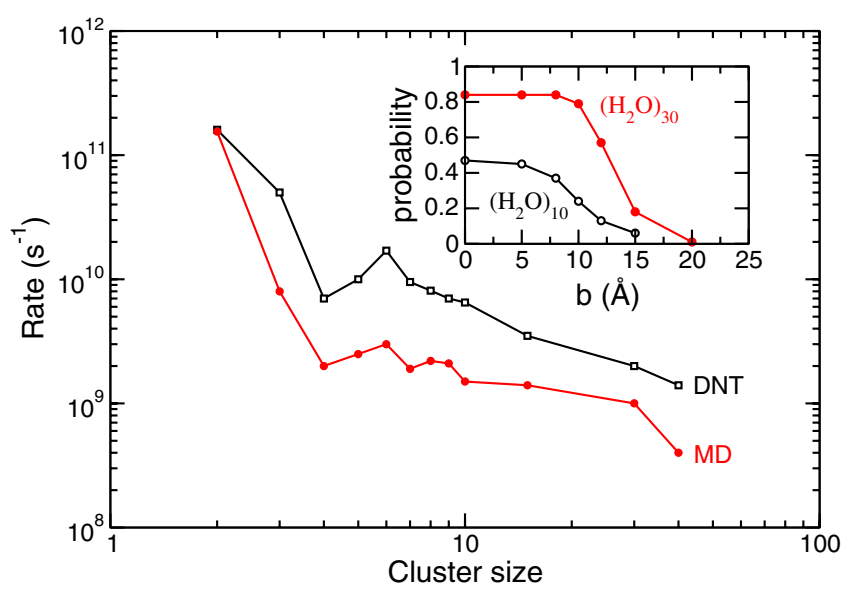

Figure 4. Evaporation rates obtained for water clusters modeled using the Dang-Chang polarizable potential from dynamical nucleation theory (empty black squares) and from direct molecular dynamics trajectories (full red circles) at $243 \mathrm{~K}$. The inset highlights the probability $p(b)$ that a water monomer sticks to water clusters thermalized at $243 \mathrm{~K}$, as a function of the impact parameber $b$. All data are taken from Ref. [64].

due to possible scattering. We show in the inset of Fig. 4 some typical results obtained by Schenter and coworkers [64] where the sticking probability $p$ of a water monomer onto two water clusters is plotted against the impact parameter $b$ of the collision. $p(b)$ never reaches 1 , even in large clusters. The soft decrease in $p$ at some value of $b$ provides another independent dynamical estimation of the cluster radius, as seen from the point of view of condensation.

Relying on the knowledge of intermolecular forces makes dynamical nucleation theory also fruitful for studying heterogeneous nucleation, and to address the sign preference issue [65]. Other efforts have tried to combine several ideas from the various theories. For instance, Reguera and Reiss [66] employed an improved expression for the free-energy $\Delta G(n)$ in CNT, a smooth droplet profile as in DFT, and the volume definition from DNT into their so-called extended modified liquid drop (EMLD) model. This model lacks any atomistic input, which makes it suitable for most substances for which the interaction potentials are not well characterized. It was found to predict accurate critical sizes and even mass distributions without additional fitting [66].

\section{EXPERIMENTS}

Systematic nucleation studies of different classes of compounds with a variety of molecular properties are important to test the role of microscopic features in homogeneous nucleation. In this section we review some important results obtained from laboratory experiments on liquid nucleation from the vapor. It should be emphasized here that such experiments are particularly hard to carry out, due to difficulties in detecting the nuclei and to control the thermodynamical parameters.

\subsection{Critical supersaturation vs. temperature}

As we have seen previously, nucleation kinetics strongly depends on the temperature $T$ and the supersaturation $S$ of the vapor, in addition to the pressure. The growth of droplets becomes very fast once the critical nucleus is formed, and this has led some experiments to measuring the critical supersaturation $S_{c}$. The determination of this quantity has been achieved by a few groups who used cloud chamber setups [67-69] in which a steady-state is reached. These experiments typically consist of two horizontal plates kept at two different temperatures, the liquid being placed on the lowest temperature plate. Solving 


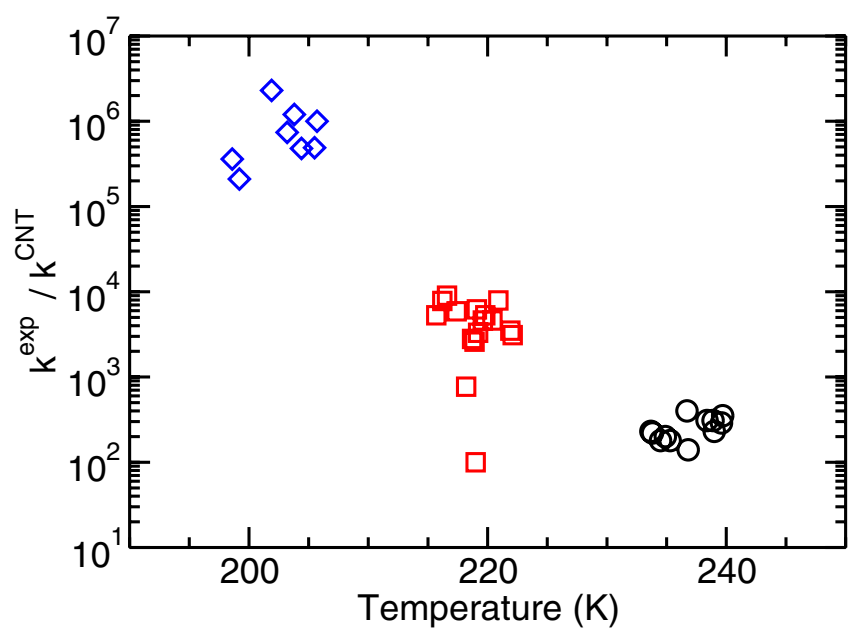

Figure 5. Ratio $k^{\exp } / k^{\mathrm{CNT}}$ between experimental and theoretical nucleation rates of $n$-nonane measured for different supersaturation ratios by Wagner and Strey (data taken from Ref. [74]), near $200 \mathrm{~K}$ (blue diamonds), $220 \mathrm{~K}$ (red squares), and $240 \mathrm{~K}$ (black circles).

the coupled energy and mass transport equations in a one-dimensional model, the vertical profiles for the temperature, partial pressure, equilibrium vapor pressure are deduced, allowing the supersaturation profile to be estimated as well. The onset of drops in the metastable vapor is monitored by light scattering using a laser beam. The nucleation process is thus analysed at some specific values of $T$ and $S$, but the nucleation rate itself is not accessed. Generally, these experimental results are relatively well reproduced using the standard models from CNT. However, experiments probing broad ranges of temperatures have shown that classical nucleation theory does not perform satisfactorily and predicts too strong variations for the critical supersaturation with temperature [69]. In addition, the level of agreement between measurements and the theory was found to be largely dependent on the molecular fluid $[68,70,71]$.

\subsection{Nucleation rate vs. supersaturation and temperature}

In the previously quoted experiments, the nucleation process was only studied under conditions of detectable rates. More recently, new experimental apparatus have been developed for measuring the nucleation rate directly, as a function of known temperature and supersaturation, and in broader conditions of detection. In thermal diffusion cloud chambers [72], nucleation kinetics can be monitored by analysing the number of drops formed in a given time window, without relying on any nucleation theory for representing the results. Several alternative protocols have also been developed in which a nucleation pulse is created during about a short time lapse of about $1 \mathrm{~ms}$ inside an expansion chamber [73-76]. In this approach, an adiabatic expansion is triggered in a vapor/carrier gas mixture and followed by some recompression, which results in some brief cooling. Upon cooling, the conditions of supersaturation are reached under millisecond time scales. Shock-tube methods [77-79], which only differ in the expansion-compression process, take advantage of the fast hydrodynamics of a gas resulting from the propagation of the shock wave inside a tube. Shorter nucleation time $(\approx 10 \mu \mathrm{s})$ are also reached in supersonic nozzles [80]. In these experiments, very high nucleation rates $\left(k \approx 10^{17} \mathrm{~cm}^{-3} \mathrm{~s}^{-1}\right)$ can be obtained at very low temperatures.

All these experiments have found evidence for markedly different dependencies for the nucleation rate as a function of temperature [74, 81] with respect to classical nucleation theory. This is illustrated in Fig. 5, where the ratio between the experimentally measured nucleation rate and its prediction between 
standard CNT, $k^{\exp } / k^{\mathrm{CNT}}$, is represented as a function of increasing temperature for $n$-nonane $\left(\mathrm{C}_{9} \mathrm{H}_{20}\right)$. The data, taken from the work by Wagner and Strey [74], are indicative of the difficulties inherent to using basic CNT, with its relatively few parameters, as an accurate template for representating experimental data.

It might be argued that these difficulties may be due to the rather complex chemical nature involved in the $\mathrm{C}_{9} \mathrm{H}_{20}$ alkane. However, comparable discrepancies between CNT and experimental data have been reported for the chemically simpler argon fluid in the temperature range $30-50 \mathrm{~K}$, CNT underestimating the experimental nucleation rates by about 11-13 orders of magnitude [82], which again suggests large discrepancies in the free energy barriers. Accurate experimental data on noble gas nucleation are particularly important because they are convenient for numerical simulations, without adding to the complexity of chemical modeling.

Besides nucleation rates, these experiments also provide information about critical nuclei, whose size appears as a crucial quantity in nucleation models. Some experiments have specifically focused on estimating $n^{*}$ by analysing how nucleation rates vary with increasing supersaturation along isotherms, estimating $n^{*}$ with the first nucleation theorem [Eq. (14)], and comparing it to the Kelvin relation, Eq. (8), using appropriate values for the surface tension and liquid density. Remaining discrepancies of $20-40 \%$ in the critical size have been reported, depending on the nature of the fluid [81-83].

One general limitation of all these experimental investigations is that nucleation is never exactly an homogeneous process due to the presence of the non-ideal carrier gas. Many authors have addressed this issue, trying to get insight into the role of the carrier gas on the clustering process by varying the total pressure [83-88]. Unfortunately, pressure effects are far from being fully understood, and experimental results are somewhat contradictory. For instance, most experiments have reported that the nucleation rate increases with decreasing total pressure, even if temperature and supersaturation ratios are both kept constant. This has been recently confirmed on measurements for the 1-propanol nucleation in helium gas [83], but is at variance with older experiments on n-butanol [87]. Needless to say, such effects induced by the environment should be taken into account when comparing experiment and theory, but they present significant additional difficulties.

\subsection{The sticking cross section issue}

One implicit assumption in classical nucleation theory lies within the prefactor of the nucleation rate, which according to standard kinetic theory depends linearly on both the molecular flux and the sticking cross section $\Sigma$ [20]. In the vast majority of nucleation studies, including standard CNT, $\Sigma$ is taken as the surface area of a hard-sphere droplet, as would be appropriate for a purely contact interaction. However, in the context of unimolecular dissociation theories based on microreversibility, the sticking cross section enters the evaporation rates as well, because it contains relevant information about the long-range interactions due to dispersion $\left(1 / r^{6}\right)$ of ion-dipole $\left(1 / r^{4}\right)$ forces. On the other hand, such reaction rate theories are valid for small molecular compounds, and neglect the spatial extension of the cluster.

Size effects on the sticking cross section have been found in molecular dynamics simulations by Venkatesh and coworkers [89], and they have been theoretically studied by Vigué and coworkers [90] in the case of large clusters bound by dispersive interactions. In this work the effective interaction between the cluster and the dissociating atom was taken as $-C_{n} /\left(r^{2}-R_{n}^{2}\right)^{3}$ with $C_{n} \propto n$ and $R_{n} \propto n^{1 / 3}$, and the calculation was carried out assuming a Langevin model. At small sizes, the long-range interaction dominates and the cross section scales as $\Sigma(n) \propto n^{1 / 3}$. Conversely, in large clusters the centrifugal barrier is very close to the cluster radius and $\Sigma(n) \propto n^{2 / 3}$, as would be expected from a pure geometrical basis.

The failure of the geometric approximation has recently been confirmed experimentally [91]. Using mass spectrometry selection, Chirot et al. have been able to measure the sticking cross section of cationic sodium clusters crossing a gas chamber of neutral sodium atoms. The variations of $\Sigma$ with size found 

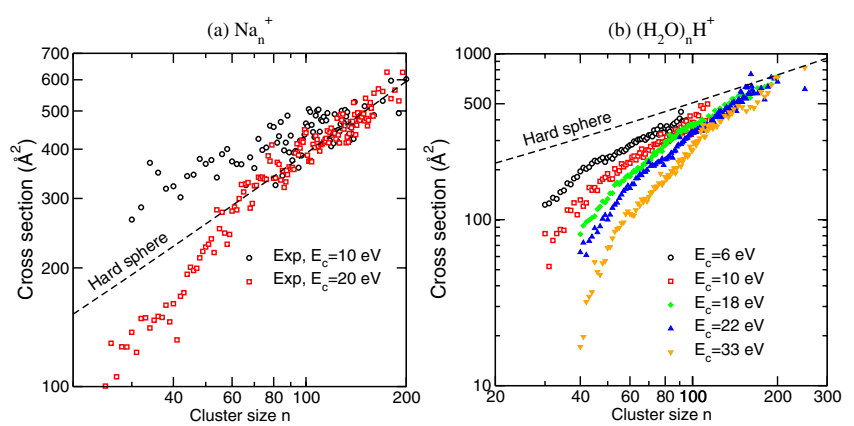

Figure 6. Variations of the sticking cross section of neutral monomers on cationic clusters, measured experimentally at various collision energies by the L'Hermite group and compared with the predictions of the simple hard-sphere model. (a) Sodium atoms on cationic sodium clusters (data taken from Ref. [91]); (b) water molecules on protonated water clusters (data taken from Ref. [92]).

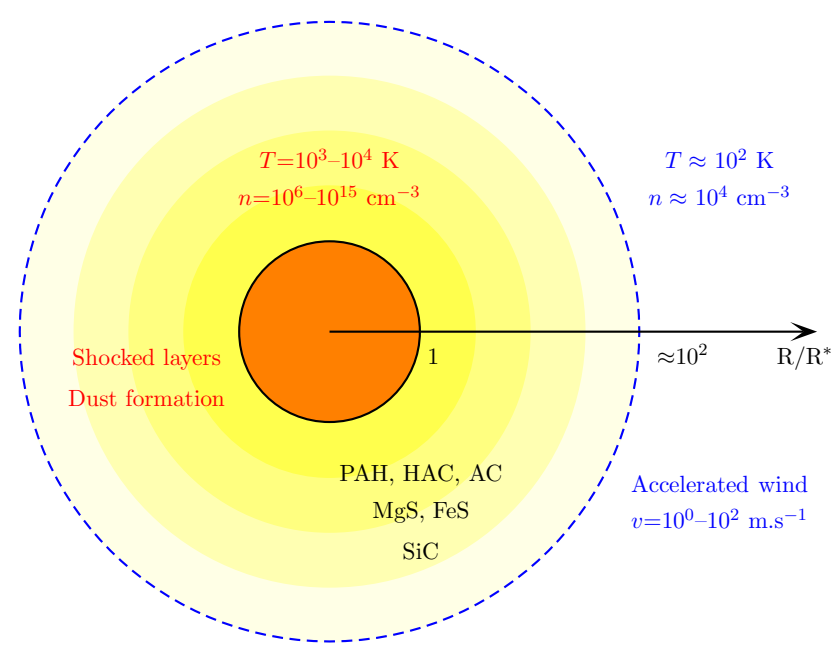

Figure 7. The main physical conditions for dust formation in the vicinity of a star.

at different collision energies, shown in Fig. 6(a), exhibit strong deviations with respect to the hard sphere model, whereas a Langevin model accounting for the smooth interaction between the cluster and the evaporating atoms performs significantly better [91]. More recently, the same group was able to extend these measurements to the important case of water impinging on cationic (protonated) water clusters [92]. The results, depicted in Fig. 6(b), indicate sticking cross sections that are lower than the hard-sphere prediction, a surprising result considering that the attractive ion-dipole interaction would predict the opposite behavior. The analysis carried out by these authors suggested inelastic scattering as a possible explanation of this effect.

\section{ASTROPHYSICAL RELEVANCE}

The presence of "pieces of solid matter" in the interstellar medium (ISM), called interstellar grains, is now well-established based on spectral features observed in absorption spectroscopy (the so-called extinction curve), as well as IR emission and light scattering properties [93]. The grain size distribution in the ISM is generally inferred from the wavelength dependence of the extinction curve, although the chemical composition is better interrogated by spectroscopy. These interstellar grains, whose 


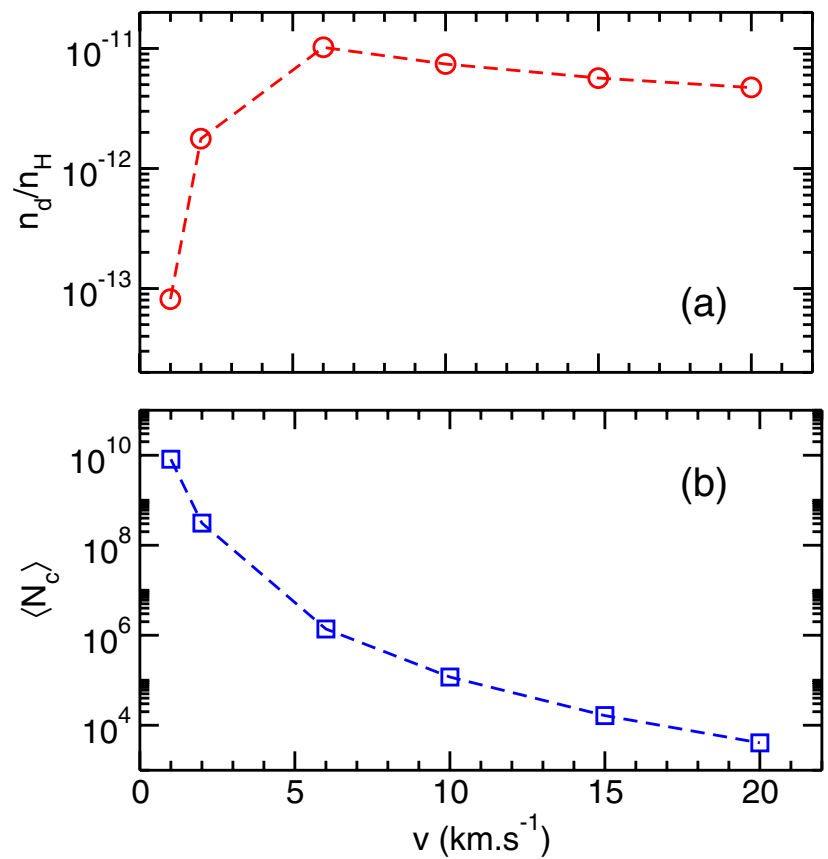

Figure 8. (a) Number of dust particles formed per hydrogen atom $n_{d} / n_{\mathrm{H}}$; (b) average number of carbon atoms $\left\langle N_{\mathrm{C}}\right\rangle$ as a function of the C-rich stellar wind velocity. The data are collected from Ref. [99].

size ranges from nanometer-scale, large molecules to $\mu \mathrm{m}$ materials, are believed to play a key role in the chemical evolution of the ISM through different mechanisms that include radiation transfer, photoelectric effects, as well as reactivity on grain surfaces. However, their composition remains debated [94]. Less controversial candidates are silicates and carbonaceous materials. The former, either in amorphous or crystalline forms, may explain the strong absorption band near $9.7 \mu \mathrm{m}$ in the extinction curve [95], very close to the typical silicate absorption around $10 \mu \mathrm{m}$ corresponding to Si-O stretchings. The carbonaceous hypothesis is mainly motivated by the very strong and broad absorption band near $2175 \AA$, referred to as the bump. Both viewpoints share a common central wavelength, but differ in the strong variations in the FWHM absorption band [96]. This bump is generally attributed to the $\pi \rightarrow \pi^{*}$ transition in graphite-like materials with $\mathrm{sp}^{2}$-bonded carbon. Due the diversity of chemical bonds and hybridization states exhibited by carbon, a broad variety of carbon-based compounds have been suggested as candidates, including diamond, graphite, amorphous carbon, but also hydrogenated amorphous carbon, large PAHs and aliphatic hydrocarbons.

There is now evidence that interstellar grains are formed in stellar outflows, and infrared emission from dust has been detected in the vicinity of carbon stars, red giants and planetary nebulae. For instance, the $10 \mu \mathrm{m}$ silicate absorption band has been detected around oxygen-rich stars $(\mathrm{O} / \mathrm{C}>1)$ [97]. However, the chemical composition of interstellar dust can strongly differ from this nascent stardust because of the various chemical and physical processes involved. Visible/UV starlight can induce photolysis or photodesorption of atoms and molecules at the grain boundaries. Interaction with cosmic rays can also damage the grain material. Inter-grain collisions and reactivity on the grain surface are other examples of processes by which composition and size are altered. All these microscopic mechanisms are related to the local environment in which dust is formed, especially at the shock wave structure around the star.

Obviously, a complete understanding of chemical composition and size distribution of interstellar dust particles requires a proper description of all alteration mechanisms at the molecular level, as well as 
some appropriate modeling of the formation kinetics. Nucleation processes have to be taken into account to describe dust growth in star outflows. However, in such dynamical environments, one major concern is the role played by out-of-equilibrium processes because, due to the outflow velocity, the growing particles follow a path in the $(P, T)$ plane around the stellar object.

A time-dependent theoretical approach was proposed by Draine and Salpeter [98] in the framework of classical nucleation theory. Based on approximate analytical solutions, this formalism provides expressions for the mean cluster size $\langle N\rangle$ as a function of time and temperature, along given density paths. The Draine-Salpeter model has been used and expanded in the astrophysical context for dust formation around stars [99]. Differential equations of the various moments of dust particles size distribution $f(N, t)$ could be established, and all the important features such as average dust size, area or radius could be expressed as a function of these moments. In the work by Gail and coworkers [99], the nucleation and growth dynamics were decoupled from the hydrodynamics, and the matter flow velocity was assumed as constant in a 1D spherical star model. In this stationary model, the pressure $P(r)$ and temperature $T(r)$ radial profiles were determined explicitlty. For given star radius and star temperature, dust formation could thus be followed as a function of the flow velocity and the mass loss rate. As an illustration, and using the original simulation data of Gail and coworkers [99], we have plotted in Fig. 8 the number of dust particles formed per hydrogen atom, $n_{d} / n_{\mathrm{H}}$, and the average number of carbon atoms $\left\langle N_{\mathrm{C}}\right\rangle$ in these dust particles as a function of the C-rich stellar wind velocity.

The above model was subsequently extended in order to couple nucleation and growth dynamics to hydrodynamics [100], and to include chemical reactivity during the growth processes [101]. Because the underlying CNT framework involves drastic approximations, and considering the impressive progresses of computational chemical physics, it now appears utterly important to revisit and question these approximations in the context of dust formation in atomic outflows around stellar objects.

\section{References}

[1] Nucleation and Atmospheric Aerosols, edited by N. Fukuta and P. E. Wagner (Deepak, Hampton, 1992)

[2] F. F. Abraham, Homogeneous Nucleation Theory (Academic, New-York, 1974)

[3] H. Richter and J. B. Howard, Prog. Energy Combust. Sci. 26, 565 (2000)

[4] M. Frenklach, Phys. Chem. Chem. Phys. 4, 2028 (2002)

[5] S. H. Chung and A. Violi, J. Chem. Phys. 132, 174502 (2010)

[6] M. Kulmala, Science 302, 1000 (2003)

[7] J. Jortner, Z. Phys. D: At. Mol. Clusters 24, 247 (1992)

[8] J. K. Lee, J. A. Barker, F. F. Abraham, J. Chem. Phys. 58, 3166 (1973)

[9] D. I. Zhukhovitskii, J. Chem. Phys. 103, 9401 (1995)

[10] R. Soto, P. Cordero, J. Chem. Phys. 110, 7316 (1999)

[11] K. Laasonen, S. Wonczak, R. Strey, A. Laaksonen, J. Chem. Phys. 113, 9741 (2000)

[12] P. Schaaf, B. Senger, J. C. Voegel, R. K. Bowles, H. Reiss, J. Chem. Phys. 114, 8091 (2001)

[13] M. Volmer, A. Weber, Z. Phys. Chem., Stoechiom. Verwandtschaftsl. 119, 277 (1926)

[14] R. Becker, W. Döring, Ann. Phys. (Leipzig) 416, 719 (1935)

[15] J. Feder, K. C. Russell, J. Lothe, G. M. Pound, Adv. Phys. 15, 111 (1966)

[16] Ya. B. Zel'dovich, Ann. Phys. (N.Y.) 18, 1 (1943)

[17] D. Kashchiev, J. Chem. Phys. 76, 5098 (1982)

[18] D. Kashchiev, J. Chem. Phys. 125, 044505 (2006)

[19] Y. Viisanen, R. Strey, H. Reiss, J. Chem. Phys. 99, 4680 (1993)

[20] I. J. Ford, Phys. Rev. E 56, 5615 (1997)

[21] B. Hale, Phys. Rev. A 33, 4156 (1986)

[22] E. Ising, Z. Physik 31, 253 (1925)

[23] S. Ryu, W. Cai, Phys. Rev. E 81, 030601(R) (2010) 
EPJ Web of Conferences

[24] D. Stauffer, A. Coniglio, D. W. Heermann, Phys. Rev. Lett. 49, 1299 (1982)

[25] P. A. Rikvold, H. Tomita, S. Miyashita, S. W. Sides, Phys. Rev. E 49, 5080 (1994)

[26] A. C. Pan, D. Chandler, J. Phys. Chem. B 108, 19681 (2004)

[27] S. Wonczak, R. Strey, D. Stauffer, J. Chem. Phys. 113, 1976 (2000)

[28] M. E. Fisher, Rep. Prog. Phys. 30, 615 (1967)

[29] C. Dellago, P. G. Bolhuis, P. Geissler, Adv. Chem. Phys. 123, 1 (2002)

[30] A. Laaksonen, V. Talanquer, D. W. Oxtoby, Annu. Rev. Phys. Chem. 46, 489 (1995)

[31] M. Blander, J. L. Katz, J. Stat. Phys. 4, 55 (1972)

[32] H. Reiss, W. K. Kegel, J. L. Katz, J. Phys. Chem. 102, 8548 (1998)

[33] S. L. Girshick, C.-P. Chu, J. Chem. Phys. 93, 1273 (1990)

[34] J. Merikanto, E. Zapadinsky, H. Vehkamäki, A. Lauri, Phys. Rev. Lett. 98, 145702 (2007)

[35] A. Dillmann, G. E. A. Meier, Chem. Phys. Lett. 160, 71 (1989)

[36] R. C. Tolman, J. Chem. Phys. 17, 333 (1949)

[37] A. Laaksonen, I. J. Ford, M. Kulmala, Phys. Rev. E 49, 5517 (1994)

[38] M. Horsch, J. Vrabec, H. Hasse, Phys. Rev. E 78, 011603 (2008)

[39] R. Evans, Adv. Phys. 28, 143 (1979)

[40] X. C. Zeng, D. W. Oxtoby, J. Chem. Phys. 94, 4472 (1991)

[41] V. Talanquer, D. W. Oxtoby, J. Chem. Phys. 100, 5190 (1994)

[42] L. Gránásy, Europhys. Lett. 24, 121 (1993)

[43] I. Kusaka, Z.-G. Wang, J. H. Seinfeld, J. Chem. Phys. 102, 913 (1995)

[44] I. Kusaka, Z.-G. Wang, J. H. Seinfeld, J. Chem. Phys. 103, 8993 (1995)

[45] C. T. R. Wilson, Phil. Trans. R. Soc. London Ser. A 189, 265 (1897)

[46] F. H. Stillinger, J. Chem. Phys. 38, 1486 (1963)

[47] L. A. Pugnaloni, F. Vericat, J. Chem. Phys. 116, 1097 (2002)

[48] S. A. Harris, I. J. Ford, J. Chem. Phys. 118, 9216 (2003)

[49] G. K. Schenter, S. M. Kathmann, B. C. Garrett, J. Chem. Phys. 110, 7951 (1999)

[50] G. K. Schenter, S. M. Kathmann, B. C. Garrett, Phys. Rev. Lett. 82, 3484 (1999)

[51] S. M. Kathmann, G. K. Schenter, B. C. Garrett, J. Chem. Phys. 111, 4688 (1999)

[52] V. Weisskopf, Phys. Rev. 52, 295 (1937)

[53] O. K. Rice, H. C. Ramsperger, J. Am. Chem. Soc. 49, 1617 (1927)

[54] L. S. Kassel, J. Phys. Chem. 32, 225 (1928)

[55] L. S. Kassel, J. Phys. Chem. 32, 1065 (1928)

[56] E. Wigner, J. Chem. Phys. 5, 720 (1937)

[57] W. H. Miller, J. Chem. Phys. 61, 1823 (1974)

[58] S. Weerasinghe, F. G. Amar, J. Chem. Phys. 98, 4967 (1993)

[59] F. Calvo, P. Parneix, J. Chem. Phys. 119, 256 (2003)

[60] F. Calvo, P. Parneix, J. Chem. Phys. 119, 9469 (2003)

[61] F. Calvo, P. Parneix, J. Chem. Phys. 121, 11088 (2004)

[62] S. M. Kathmann, G. K. Schenter, B. C. Garrett, J. Chem. Phys. 116, 5046 (2002)

[63] L. D. Crosby, S. M. Kathmann, T. L. Windus, J. Comput. Chem. 30, 743 (2009)

[64] G. K. Schenter, S. M. Kathmann, B. C. Garrett, J. Chem. Phys. 116, 4275 (2002)

[65] S. M. Kathmann, G. K. Schenter, B. C. Garrett, Phys. Rev. Lett. 94, 116104 (2005)

[66] D. Reguera, H. Reiss, Phys. Rev. Lett. 93, 165701 (2004)

[67] G. Agarwal, R. H. Heist, J. Chem. Phys. 73, 902 (1980)

[68] D. Kane, M. Samy El-Shall, J. Chem. Phys. 105, 7617 (1996)

[69] M. M. Rudek, J. L. Katz, H. Uchtmann, J. Chem. Phys. 110, 11505 (1999)

[70] M. S. El-Shall, J. Chem. Phys. 90, 6533 (1989)

[71] D. Wright, R. Caldwell, C. Moxely, M. S. El-Shall, J. Chem. Phys. 98, 3356 (1993)

[72] C. -H. Hung, M. J. Krasnopoler, J. L. Katz, J. Chem. Phys. 90, 1856 (1989)

[73] J. L. Schmitt, R. A. Zalabsky, G. W. Adams, J. Chem. Phys. 79, 4496 (1983) 
[74] P. E. Wagner, R. Strey, J. Chem. Phys. 80, 5266 (1984)

[75] A. Fladerer, R. Strey, J. Chem. Phys. 124, 164710 (2006)

[76] K. Iland, J. Wölk, R. Strey, D. Kashchiev, J. Chem. Phys. 127, 154506 (2006)

[77] F. Peters, B. Paikert, J. Chem. Phys. 91, 5672 (1989)

[78] A. Graßmann, F. Peters, J. Chem. Phys. 113, 6774 (2000)

[79] C. C. M. Luitjen, K. J. Bosschaart, M. E. H. van Dongen, J. Chem. Phys. 106, 8116 (1997)

[80] Y. J. Kim, B. E. Wyslouzil, G. Wilemski, J. Wölk, R. Strey, J. Chem. Phys. 108, 4365 (2004)

[81] D. Brus, V. Zdimal, J. Smolik, J. Chem. Phys. 129, 174501 (2008)

[82] S. Sinha, A. Bhabhe, H. Laksmono, J. Wölk, R. Strey, J. Chem. Phys. 132, 064304 (2010)

[83] D. Brus, V. Zdimal, F. Stratmann, J. Chem. Phys. 124, 164306 (2006)

[84] J. Franck, H. G. Hertz, Z. Phys. 143, 559 (1956)

[85] V. Zdimal, J. Smolik, H. Uchtmann, J. Aerosol Sci. 26, 625 (1995)

[86] R. H. Heist, M. Janjua, J. Ahmed, J. Phys. Chem. 98, 4443 (1994)

[87] Y. Viisanen, R. Strey, J. Chem. Phys. 101, 7835 (1994)

[88] M. P. Anisimov, K. M. Anisimov, I. V. Gribanov, R. Y. Zamarayev, N. N. Nalimova, Y. I. Povolyaev, Colloid J. 56, 407 (1994)

[89] R. Venkatesh, R. R. Lucchese, W. H. Marlow, J. Schulte, J. Chem. Phys. 102, 7683 (1995)

[90] J. Vigué, P. Labastie, F. Calvo, Eur. Phys. J. D 8, 265 (2000)

[91] F. Chirot, P. Labastie, S. Zamith, J.-M. L'Hermite, Phys. Rev. Lett. 99, 193401 (2007)

[92] S. Zamith, P. Feiden, P. Labastie, J.-M. L'Hermite, Phys. Rev. Lett. 104, 103401 (2010)

[93] B. Draine, Annu. Rev. Astron. Astrophys. 41, 241 (2003)

[94] A. Blain, F. Combes, B. Draine in The Cold Universe, Saas-Fee Advanced Course 32, edited by D. Pfenniger and Y. Revaz, Springer (2002)

[95] F. Molster, C. Kemper, Space Sci. Rev. 119, 3 (2005)

[96] E. L. Fitzpatrick, D. Massa, Astrophys. J. 307, 286 (1986)

[97] W. C. Danchi, M. Bester, C. G. Degiacomi, L. J. Greenhill, C. H. Townes, Astrophys. J. 107, 1469 (1994)

[98] B. Draine, E. E. Salpeter, J. Chem. Phys. 67, 2230 (1977)

[99] H. P. Gail, R. Keller, E. Sedlmayr, Astron. Astrophys. 133, 320 (1984)

[100] H. P. Gail, E. Sedlmayr, Astron. Astrophys. 171, 197 (1987)

[101] H. P. Gail, E. Sedlmayr, Astron. Astrophys. 206, 153 (1988) 